\title{
7 RETOS DE LAS UNIVERSIDADES CATÓLICAS ECUATORIANAS ANTE LA TRANSFORMACIÓN DE LA EDUCACIÓN SUPERIOR EN EL ECUADOR
}

Dr. Fernando Ponce León, SJ

Profesor titular de la Pontificia Universidad Católica del Ecuador (PUCE)

\section{Resumen}

En la sociedad contemporánea el conocimiento es un bien decisivo. Pero este bien ha sido utilizado de muchas maneras, no siempre para la conservación de la vida en el planeta. Es una responsabilidad de las universidades el reconectar conocimiento y vida.

Las universidades católicas tienen mucho que aportar en este sentido, mediante una oferta educativa con determinadas características. El presente artículo sintetiza el pensamiento católico sobre conocimiento y educación superior en seis tesis. A continuación presenta tres conclusiones para el compromiso de las universidades católicas en el Ecuador, que se pueden resumir así: a) la consideración del desarrollo integral y sostenible como horizonte de sentido para la educación superior y sus tres funciones sustantivas; b) la comprensión del conocimiento como un bien público y de la educación superior como un derecho y deber de la sociedad civil, un servicio tanto estatal como civil, al tiempo que se defiende la diversidad de ofertas de sentido en educación, porque diversa es la sociedad ecuatoriana; c) la inclusión educativa como dinámica que reúne calidad y acceso democrático a la educación superior.

Palabras clave: Universidad católica, conocimiento, bien público, educación superior. 


\title{
CHALLENGES FOR THE ECUADORIAN CATHOLIC UNIVERSITIES IN THE FACE OF THE TRANSFORMATION OF HIGHER EDUCATION IN ECUADOR
}

\begin{abstract}
In contemporary society, knowledge is a fundamental good. But this good has been used in many ways, not always for the conservation of life on the planet. It is the responsibility of universities to reconnect knowledge and life. Catholic universities have much to contribute in this regard through educational opportunities with certain characteristics. This article synthesizes Catholic thought on knowledge and higher education in six concepts. The following are three conclusions regarding the commitment of Catholic universities in Ecuador. These can be summarized as follows: a) the consideration of integral and sustainable development as a framework for constructing meaning for higher education and its three substantive functions. b) The understanding of knowledge as a public good, and of higher education as a right and responsibility of civil society; a service from both the state and civil society, while defending the diversity of opportunities of meaning in education because the Ecuadorian society is diverse. c) Educational inclusion as a dynamic that brings quality and democratic access to higher education.
\end{abstract}

Keywords: catholic university, knowledge, public good, higher education 


\section{INTRODUCCIÓN}

Se cuenta que al principio de los tiempos Dios plantó en el jardín del Edén el árbol del conocimiento y el árbol de la vida. Entregó al hombre todo el jardín para que "lo cultivara y lo guardara" (Génesis 2, 15), con una sola condición: que no tocara ninguno de los dos árboles porque el apropiárselos le acarrearía nefastas consecuencias. Sin embargo, el hombre cedió a la tentación, comió del fruto del árbol del conocimiento y Dios lo expulsó del paraíso, junto con su compañera. No solo fue un castigo a la infracción del mandato divino, sino una sabia medida preventiva para que el hombre no alcanzara el otro árbol, el árbol de la vida, y terminara así por arruinar toda la creación.

Este mito judeo cristiano describe nuestra situación actual con mucha agudeza. Los humanos hemos usado y abusado del conocimiento, la ciencia y la técnica, y nos hemos endiosado creyendo conocer absolutamente todo sobre todo. Hemos aprendido a destruir - piénsese en las bombas atómicas que arrasaron Hiroshima y Nagasaki-, pero todavía no sabemos cómo proteger adecuadamente las diversas formas de vida que con igual derecho pueblan el planeta. Si no cambiamos nuestra manera de utilizar los diversos conocimientos que producimos, el árbol de la vida terminará "hecho leña", como decimos coloquialmente.

Esta situación plantea una enorme responsabilidad a las universidades Su misión tradicionalmente se ha descrito como la búsqueda y comunicación de la verdad. Actualmente se expresa como el avance de los conocimientos, la ciencia y la técnica a fin de responder a retos mundiales, como la seguridad alimentaria, el cambio climático, la gestión del agua, el diálogo intercultural, las energías renovables, la salud pública, la reducción de la pobreza, entre otros (UNESCO / Conferencia Mundial sobre Educación Superior 2009, 2010, pág. 5). Hoy más que nunca, necesitamos reanudar el nexo entre conocimiento y vida.

En este artículo quisiera explorar cómo las universidades católicas pueden aportar a esta gran responsabilidad. Estas instituciones son universidades como las demás, sometidas a los mismos desafíos y presiones que cualquiera de ellas. Al mismo tiempo, la motivación de su quehacer académico proviene de la cosmovisión católica sobre la persona y la sociedad y, en último término, de la vida y obra de Jesús de Nazareth. Parto de la hipó- 
tesis de que este enfoque particular tiene mucho que contribuir en las transformaciones por las que está pasando la educación superior en el Ecuador.

Debido a razones de espacio me limitaré a señalar seis rasgos generales de lo que considero es el pensamiento de la Iglesia sobre el conocimiento y la educación superior, para luego deducir tres conclusiones sobre el problema enunciado: la correcta articulación entre conocimiento y mejores condiciones de vida, en el contexto particular del Ecuador.

Lo que sigue es una elaboración muy personal. La Iglesia Católica dispone de un documento propio sobre las universidades católicas, la Constitución Apostólica Ex Corde Ecclesiae de san Juan Pablo II, que se puede consultar para conocer el pensamiento oficial al respecto. Tomándolo como base, y apoyándome en otras fuentes, procuraré presentar una reflexión argumentada, razonable y defendible, que pueda entrar en debate con otras posturas sobre la apasionante y urgente cuestión de la responsabilidad social de la educación superior en nuestro país. 


\title{
PENSAMIENTO CATÓLICO SOBRE EL CONOCIMIENTO Y LA EDUCACIÓN
}

\author{
A riesgo de simplificar, diré que el pensamiento social \\ católico sobre el conocimiento y la educación puede resu- \\ mirse en seis afirmaciones.
}

En primer lugar, la Iglesia da por hecho que el conocimiento es una nueva forma de riqueza, como se afirma en todas las discusiones sobre la sociedad del conocimiento. En su encíclica Centesimus Annus, Juan Pablo II hace un análisis de la situación mundial luego de la caída del muro de Berlín. Al analizar el capitalismo sostiene que este sistema no resulta éticamente justificado solo por el hecho de emerger como triunfador de la guerra fría. Luego añade: "Existe otra forma de propiedad, concretamente en nuestro tiempo, que tiene una importancia no inferior a la de la tierra: es la propiedad del conocimiento, de la técnica y del saber. En este tipo de propiedad, mucho más que en los recursos naturales, se funda la riqueza de las naciones industrializadas" (Juan Pablo II, 1991, n. 32).

En efecto, el conocimiento es un factor generador de riqueza personal y social en la sociedad contemporánea porque, como dice un experto mexicano, "Ios conocimientos ya no sólo se generan y se transmiten como antaño, sino que hoy en día se registran, se aplican, se patentan, se comercializan, se asocian, se exportan, se importan." (De La Fuente, 2008, pág. 26).
Por este motivo, el Estado y las empresas en los países desarrollados reconocen que la inversión en educación superior es una movida estratégica para volver más competitivas sus economías nacionales y atraer inversiones, algo que no sucede en América Latina, según el mismo experto.

En segundo lugar, el valor principal del conocimiento y de la educación radica en su contribución al crecimiento y plenitud de la persona. Del conocimiento se pueden decir muchas cosas: que es un factor de riqueza, como se mencionó, que permite el ascenso social de sus poseedores; o que constituye el patrimonio de un país, cuando se consideran los productos concretos en relación con un colectivo culturalmente homogéneo. Sin embargo, más allá de estos valores instrumentales, para el pensamiento social católico y otras filosofías humanistas, el conocimiento en sus múltiples formas es valioso sobre todo porque contribuye al desarrollo de la persona en su integralidad, puesto que fomenta el espíritu crítico, la aproximación a la verdad, el sentido de la historia y las tradiciones. El conocimiento humaniza, además de ser útil. 
Lo mismo puede decirse de la educación. En rigor, conocimiento y educación no son lo mismo; esta es un proceso o conjunto de procesos propios de una sociedad, que permite a las personas el desarrollo de sus habilidades y la asimilación de conocimientos, o aprendizaje, dicho de manera muy general. Sin embargo, para el caso que nos ocupa, el principal valor de la educación, incluida la educación superior, es también su contribución al desarrollo humano integral de la persona, antes que su utilidad social o económica. Por la misma razón que es valioso lo que se asimila, es valioso el proceso que conduce a esa asimilación, al aprendizaje. Incluso si se destacan en la educación sus aspectos no cognitivos, como por ejemplo el desarrollo emocional de quien aprende o de sus capacidades relacionales, también se llegará a la misma conclusión sobre su valor humanizante.

En este sentido, el pensamiento católico concuerda bien con afirmaciones como las de Amartya Sen, Premio Nobel de economía en 1999, quien se pregunta: "¿Qué hace el desarrollo humano? La creación de oportunidades sociales ofrece una contribución directa a la expansión de las capacidades humanas y la calidad de vida. La expansión de los cuidados de salud, educación, seguridad social, etc. contribuyen directamente a la calidad de vida y a su florecimiento" (Sen, 1999, pág. 144). Esta manera de entender la educación y el conocimiento como posibilitantes de personas capaces y libres para vivir una vida razonablemente elegida, es substancialmente diferente, aunque no contrapuesta, a esa otra que los entiende como herramientas esenciales para aumentar la productividad de personas y sociedades: la teoría del capital humano.

En tercer lugar, la generación, difusión y aplicación del conocimiento implican exigencias éticas, y con mayor razón su registro y comercialización. Ya se dijo esto más arriba cuando se mencionó la responsabilidad de las universidades ante los grandes retos mundiales. Al hablar sobre el desarrollo humano integral, el anterior, Papa Benedicto XVI, sostiene: "... el tema del desarrollo humano integral adquiere un alcance aún más complejo: la correlación entre sus múltiples elementos exige un esfuerzo para que los diferentes ámbitos del saber humano sean interactivos, con vistas a la promoción de un verdadero desarrollo de los pueblos. Con frecuencia, se cree que basta aplicar el desarrollo o las medidas socioeconómicas correspondientes mediante una actuación común. Sin embargo, este actuar común necesita ser orientado, 
porque "toda acción social implica una doctrina»" (Benedicto XVI, 2009, n. 30).

En otras palabras, el horizonte ético del conocimiento está determinado en última instancia por una "doctrina" o ideología sobre la sociedad en la que queremos vivir. En el pensamiento social de la Iglesia este horizonte viene dado por su articulación con el desarrollo humano integral, en palabras de Benedicto XVI, o con el desarrollo integral y sostenible, según la enseñanza social renovada por el actual pontífice (Francisco, Carta Encíclica Laudato Si. Sobre el cuidado de la casa común, 2015). Este horizonte ético compagina bien con el concepto de desarrollo que sustentan los Objetivos de Desarrollo Sostenible de la ONU, especialmente con el objetivo cuarto, referido a la educación, y su tercera meta, relacionada con la educación superior, aunque mantenga ciertas diferencias con algunos de los planteamientos onusianos.

En cuarto lugar, la educación es un componente esencial del bien común, lo cual también puede decirse de los conocimientos que la educación permite alcanzar. Como se sabe, el bien común es uno de los principios rectores de la ética social del catolicismo. Según este enfoque ético, el bien común no significa lo mismo que el bien total, que es la suma de los bienes propios de los individuos, sino que se refiere al bien de la comunidad en cuanto sujeto colectivo distinto de los sujetos individualmente considerados y sumados. Según la comprensión clásica de bien común, lo nuevo que aparece en el sujeto colectivo, y cuya realización o garantía constituye lo nuclear de esta noción, son las condiciones generales que posibilitan los bienes propios o individuales. Así lo entiende el Concilio Vaticano II cuando define al bien común como "el conjunto de condiciones de la vida social que hacen posible a las asociaciones y a cada uno de sus miembros el logro más pleno y más fácil de la propia perfección". (Concilio Vaticano II, 1965, n. 26). En este sentido, la educación es una condición general y esencial para la vida plena de personas y asociaciones de la sociedad civil.

Según otra interpretación igualmente válida lo esencialmente distintivo de una comunidad respecto de un conglomerado de individuos serían las relaciones entre individuos que por sí mismas son constitutivas de una comunidad, no las condiciones que facilitan la realización humana de personas o asociaciones. Así, el bien común debería decirse de las relaciones como fin último de la comunidad, no de las condiciones generales, que no serían sino 
instrumentos o medios para los bienes propios (Zamagni, 2007). Como quiera que sea, la consecuencia importante es que la educación, incluida la educación superior, es obligación del Estado. Ya sea porque la educación condiciona la realización personal y de las asociaciones, o porque modela las relaciones en el interior de una comunidad, corresponde al Estado su plena garantía, a condición que este tenga por finalidad primordial la promoción del bien común, como lo sostiene el pensamiento social católico y otros enfoques en filosofía política.

Valga la pena señalar que el Papa Francisco introduce en la comprensión del bien común tres nuevos elementos (Caamaño López, 2016). Primero, este principio no abarca únicamente las condiciones sociales, sino más integralmente las condiciones socio-ambientales que permiten la vida en plenitud. Segundo, la preocupación por el bien común implica una preocupación por la justicia distributiva desde la óptica de la opción por los excluidos. Por último, esta justicia distributiva debe considerar también las generaciones futuras, no solo las presentes.

En quinto lugar, el conocimiento es un bien público. Desde el punto de vista de los economistas, "bien público", o su sinónimo "bien colectivo" (Laville \& Cattani, 2006) (Ricossa, 2000) es un tipo especial de bien de consumo distinto del bien privado. Un bien público, en este sentido especializado, tiene dos características: la no rivalidad, que significa que su consumo por parte de una persona no impide el consumo por parte de otra; y la no exclusión, que quiere decir que resulta imposible o excesivamente costoso excluir a alguien del consumo de ese bien (Stiglitz, 1999, pág. 308). Así, por ejemplo, el alumbrado de las calles no mengua si muchas personas se benefician de él, y sería prácticamente imposible impedir que uno o algunos se beneficien de este servicio, que con razón se llama comúnmente servicio público.

Debido a estas características los bienes públicos no pueden ser garantizados ni producidos por el mercado, porque no resultan rentables. Esto hace que el Estado deba intervenir para su producción y distribución. En este punto hay que notar que lo público no es equivalente a lo estatal. Por un lado, un bien público es tal debido a sus características esenciales, no porque el Estado se haga responsable de él; el carácter de público de un bien antecede a su carácter estatal. Por otra parte, un Estado bien puede responsabilizarse de bienes privados por razones estratégicas; por ejemplo, de los alimentos o los combustibles. De aquí 
que no todo bien estatal sea necesariamente un bien público en este sentido especializado.

El conocimiento es un bien público en este sentido. El que más personas sepan más, en cualquier ámbito del saber, no disminuye en nada la posibilidad de que otras personas también lo hagan. La difusión del conocimiento y la libertad de una persona para conocer no merman los conocimientos adquiridos por otras personas ni el acervo cultural y científico de la humanidad.

Así lo afirma el pensamiento de la Iglesia. En su comentario sobre los bienes, cuya defensa y tutela corresponde al Estado en el nuevo capitalismo posterior al año 1989, el Papa Juan Pablo II sostiene que el mercado tiene sus límites: "existen necesidades colectivas y cualitativas que no pueden ser satisfechas mediante sus mecanismos; hay exigencias humanas importantes que escapan a su lógica; hay bienes que, por su naturaleza, no se pueden ni se deben vender o comprar" (Juan Pablo II, 1991, n. 40). El conocimiento es indudablemente uno de estos bienes que por su naturaleza y su función no pueden ser tratados como mercancía.

Pero ¿qué sucede con la educación superior? Parece que no cabe decir que este mecanismo de acceso a conocimientos de un cierto tipo sea un bien público en el sentido utilizado porque su "consumo" sí genera costos. La educación en cuanto tal no cumple la condición de no rivalidad porque el acceso de unas personas a la educación sí limita el acceso de otros a este bien. Todos los insumos que intervienen en los procesos educativos son escasos y por tanto tienen costos. Por otra parte, la educación tampoco cumple la condición de no exclusión. No es verdad que sea imposible o excesivamente costoso impedir que alguien acceda a este bien; al contrario, lamentablemente es fácil y sucede todo el tiempo. De hecho, si más personas acceden a la educación, en cualquiera de sus niveles, sí habrá disminución de posibilidades de acceso para otras personas, simplemente por razones logísticas y pragmáticas. En tal caso, el acceso a la educación pareciera ser un bien semi-público, como dice Amartya Sen (Sen, 1999, pág. 128).

Sin embargo, es común que se hable de la educación como un bien público para significar que la educación es un derecho de todos y un deber del Estado (Dias Sobrinho, 2008). Es verdad que la educación es esto y mucho más, pero este uso estratégico del término no debe confundirse con su uso especializado, como se 
ha explicado. Un ejemplo de esto puede verse en el documento oficial de los obispos latinoamericanos reunidos en su quinta conferencia general en Aparecida, Brasil. Este documento dedica tres párrafos al análisis de la "educación como bien público" con el fin de insistir en la responsabilidad del Estado respecto de la educación religiosa en las instituciones educativas estatales ( $V$ Conferencia General del Episcopado Latinoamericano y del Caribe, 2007, págs. 233-234). Quizás lo más adecuado sería decir que la educación es un servicio, antes que un bien, y un servicio estatal antes que un servicio público, como se explicará más abajo.

Las afirmaciones anteriores llevan a la sexta: la educación es un derecho humano. Si el conocimiento trae diversos beneficios individuales y sociales, al punto que constituye condición esencial para una vida plena, resulta natural que la educación, vía de acceso al conocimiento, sea considerada una exigencia cuyo cumplimiento todos los ciudadanos pueden reclamar es decir, un derecho. Un pensamiento igualitario como el cristianismo no puede pensar distinto ni aceptar que este bien quede al alcance solo de quienes pueden pagarlo.

El Concilio Vaticano II así lo afirma cuando, en su declaración sobre la educación cristiana, dice al inicio que "todos los hombres, de cualquier raza, condición y edad, en cuanto partícipes de la dignidad de la persona, tienen el derecho inalienable a una educación que responda al propio fin, al propio carácter, al diferente sexo, y que sea conforme a la cultura y las tradiciones patrias, y, al mismo tiempo, esté abierta a las relaciones fraternas con otros pueblos a fin de fomentar en la tierra la verdadera unidad y la paz" (Concilio Vaticano II, 1965b, n. 1). De manera mucho más directa, el Papa Juan Pablo II incluye explícitamente en su lista de derechos humanos "el derecho a madurar la propia inteligencia y la propia libertad a través de la búsqueda y el conocimiento de la verdad" (Juan Pablo II, 1991, n. 47). 


\section{TRES CONSECUENCIAS PARA LAS UNIVERSIDADES CATÓLICAS ECUATORIANAS ${ }^{1}$}

\section{1.- LA FUENTE IDEOLÓGICA DE LAS UNIVERSIDADES CATÓLICAS}

"Toda acción social implica una doctrina", dice Benedicto XVI citando a Paulo VI. Considerando la presente crisis socio-ambiental mencionada al inicio, y dado que la educación superior es una acción de enorme influjo en la sociedad, la fuente doctrinal para las universidades católicas del Ecuador y del mundo debería ser hoy la encíclica Laudato Si del Papa Francisco, que guarda armonía con los objetivos de desarrollo sostenible de la comunidad internacional.

Bien sabemos que no cabe tomar el documento papal como un documento técnico, ni interpretarlo como estudio de pertinencia o un plan estratégico, aunque incluya sólidos fundamentos científicos y orientaciones prácticas. La Laudato Si es una seria invitación a pensar las instituciones católicas de educación superior como universidades enfocadas en el desarrollo integral y sostenible del país y del mundo.

Admitamos que las ideologías universitarias existen. No hay duda que todas las instituciones universitarias, y en general toda organización social, suponen un determinado diagnóstico del mundo, junto con una cierta idea del mundo deseable y de los medios para alcanzarlo, tríada de elementos que constituyen una ideología, un término que aquí carece de connotación valorativa. Las ideologías universitarias generalmente se encuentran implícitas en sus declaraciones y actividades. Hay que rastrearlas en las prioridades que dan a valores como el concepto de educación superior, el éxito individual, la libertad para emprender, la perti-

$1 \quad$ Cinco universidades ecuatorianas forman parte de la Organización de Universidades Católicas de América Latina (ODUCAL), filial regional de la Federación Internacional de Universidades Católicas (FIUC). Son la Pontificia Universidad Católica del Ecuador, la Universidad Politécnica Salesiana, la Universidad Particular de Loja, la Universidad del Azuay y la Universidad Católica de Santiago de Guayaquil. Por otra parte, dos universidades adicionales reconocen expresamente que se fundamentan en los principios cristianos. Son la Universidad Católica de Cuenca y la Universidad de los Hemisferios. De manera que siete universidades ecuatorianas se inspiran en los principios cristianos católicos y son, en este sentido, universidades católicas. 
nencia, el desarrollo del país, porque difícilmente la encontraremos expuestas a la luz del día.

Las universidades que se adhieren a la cosmovisión católica, independientemente de sus formas y diversos grados de adhesión y compromiso con esta cosmovisión, no tienen que buscar lejos la fuente para construir su visión ideológica, en el sentido neutro del término. En julio de 2015, en la sede de la PUCE en Quito, el Papa Francisco confrontó a los educadores del país, y por tanto a las universidades católicas, con dos preguntas que todavía guardan vigencia: "¿Dónde está tu hermano?" "¿Para qué nos necesita esta tierra?" (Francisco, Encuentro con el mundo de la enseñanza. Discurso del Santo Padre, 2015b) Esta tierra, que es naturaleza y pueblo, ¿qué nos pide? Estos son precisamente los ejes de la encíclica a la cual nos referimos: la reconciliación social y la reconciliación ambiental, dos dimensiones de una sola misión².

\section{2.- BIEN PÚBLICO GESTIONADO POR LA SOCIEDAD CIVIL}

El conocimiento es un bien público, en el sentido ya explicado. Sin embargo, el acceso a la educación plantea dificultades porque implica distribuir recursos escasos, como espacio físico, becas, horas de profesores, entre un gran número de aspirantes. últimamente hemos visto que, más allá de las buenas intenciones gubernamentales, no existe capacidad real para garantizar a todo el que lo desee el acceso a la educación superior, de manera gratuita.

Si aceptamos entonces que el conocimiento es bien público, no sujeto a compra y venta, y a la vez constatamos que el Estado no puede garantizar como debiera la educación superior, que permite la generación y aprendizaje de conocimientos, debe aceptarse la participación de la sociedad civil como oferente de educación superior. Esto es muy diferente a admitir o tolerar la existencia de universidades particulares, que yo prefiero llamarlas universidades de la sociedad civil, solo por la imposibilidad fáctica de eliminarlas.

$2 \quad$ Lo dicho en esta sección debería completarse con una reflexión sobre cómo ser institución de inspiración cristiana en una sociedad secular como la ecuatoriana. De hecho, todas las universidades católicas del mundo enfrentan el mismo desafío de repensarse en la civilización secular contemporánea. Un congreso de educación católica organizado por el Vaticano en noviembre de año 2015 hizo de la relación entre identidad y misión uno de los temas principales de debate (Congregación para la educación católica, 2015). Lamentablemente esto quedará para otra ocasión no por menos importante sino por falta de espacio. 
Hay dos razones adicionales para esto, además de las insuficiencias del servicio educativo estatal. En primer lugar, la sociedad civil es anterior al Estado. Este nace de aquella para convertirse en comunidad organizada para la búsqueda del bien común y la garantía de los bienes públicos. Pero esto no quiere decir que la sociedad civil, a través de sus asociaciones, renuncia al derecho esencial que le corresponde de buscar el bien común y garantizar los bienes públicos. Si bien las asociaciones de la sociedad civil son espacios de sociabilidad en torno a intereses propios, en ciertos casos existen organizaciones civiles que también buscan el bien común, no solo el bien de los asociados, y una de estas es la Iglesia católica. Hay que repetirlo una y otra vez: lo público no se agota en lo estatal; lo público supera a lo estatal, de modo que es más exacto decir que el conocimiento es un bien público, y la educación es un servicio estatal, así como puede ser un servicio civil. Lastimosamente "público" ha venido a ser un sinónimo de "estatal" en el lenguaje ordinario, y esto desdibuja la comprensión tanto de los bienes públicos como de los servicios estatales.

La segunda razón en defensa de la provisión particular de este bien público que se llama conocimiento se basa en tres derechos, cuya garantía ha costado mucho en nuestra historia republicana y que ahora están reconocidos entre los artículos 26 a 29 de la Constitución: el derecho de toda persona a la educación, el derecho a la libertad de enseñanza, que corresponde a personas y entidades de la sociedad civil, y el derecho a la libertad de los padres de escoger para sus hijos la educación más acorde con sus convicciones que, entiendo yo, también se aplica a la educación superior.

En otras palabras, el acceso a la educación superior es, en teoría, para todos, pero en la práctica no alcanza para todos. Si a esto sumamos las tres libertades mencionadas, podemos afirmar lo siguiente. Incluso si el Estado tuviera ingentes recursos para garantizar a todos los jóvenes ecuatorianos el acceso universal y gratuito a la educación superior hasta el tercer nivel - y ojalá que algún día lleguemos a esto - todavía deberá contar con las instituciones de la sociedad civil para la provisión de educación superior como medio para garantizar la adquisición de conocimientos. La diversidad de ofertas en educación superior es buena en sí y es buena para la democracia, además de ser un derecho de toda persona y de toda entidad de la sociedad civil. 


\section{3.- CALIDAD E INCLUSIÓN}

Al ser el conocimiento un valor que contribuye al desarrollo personal, un bien público cuyo "consumo" por unos no perjudica a otros, un componente esencial del bien común y un derecho, es un deber ético del Estado y la sociedad civil garantizar el acceso a la educación superior a la mayor cantidad posible de personas, idealmente a todos. Ahora bien, el mayor acceso siempre trae a debate la cuestión de la calidad educativa. Se piensa que esta disminuye si más personas acceden a la educación superior. Pero la respuesta no consiste en restringir el acceso de todos a fin de "salvar" la calidad educativa de pocos, sino justamente ofrecer educación de calidad a todos, sobre la base de la igualdad de oportunidades en el acceso. La respuesta está en la inclusión educativa de calidad.

Debe señalarse que la inclusión no está reñida con la calidad ni con la excelencia en educación. Al respecto pueden identificarse tres posturas sobre la relación entre inclusión y calidad. En primer lugar, está la postura elitista. Según esta, la calidad educativa importa, lo cual nadie disputará. Pero como no todos los aspirantes a la universidad vienen con las mismas capacidades, y además no hay suficientes recursos estatales para garantizar la igualdad de oportunidades de todos, no queda más que permitir que acceda solo la elite académica, se nos dice, porque admitir a todos llevaría a rebajar la calidad de la institución que los recibe. Para esta postura, la calidad de una institución educativa depende en gran medida de la calidad de los estudiantes que ingresan. Si los mejores ingresan, supuestamente la calidad de la institución se mantiene alta. En consecuencia, la inclusión queda condicionada por la calidad que se desea mantener.

En segundo lugar, la postura masificadora pone el énfasis en el acceso para todos. Para esta postura la calidad importa menos que el acceso. Todos deberían entrar a la educación superior, porque la educación es un derecho, aunque no todos puedan acceder a una educación de calidad. La calidad es un discurso burgués y elitista que solo persigue la permanencia del status quo, dice esta postura.

Ambas posturas, por diversas que parezcan, comparten el supuesto que calidad educativa es equivalente a elitismo. En el primer caso se lo defiende, en el segundo se lo denosta; pero en ambos casos la calidad educativa se comprende como un estado 
acabado del aspirante y del estudiante y, por consiguiente, de las instituciones que los reciben.

La tercera postura podría llamarse democrática o incluyente. Para esta visión, la calidad importa, pero no como estado acabado del aspirante a la universidad ni como requisito de entrada a la universidad. La calidad educativa es un objetivo a alcanzar mediante un proceso que debe iniciarse desde la primera infancia, y que en las instituciones de educación superior comprende los cursos de nivelación, las tutorías integrales, la diversificación de ofertas de educación superior, entre otras estrategias. En este sentido, la calidad de una institución no significa seleccionar a los mejores sino conducir a todo estudiante a su mayor grado posible de excelencia académica. Según esta concepción dinámica, la calidad de una institución no se mide por la excelencia académica de los estudiantes que ingresan - lo propio de las instituciones llamadas "de elite" y que hace fantasear a no pocos mercadólogos universitarios - sino por la excelencia académica con la que egresan.

\section{CONCLUSIÓN}

En resumen, el aporte de las universidades católicas al proceso de transformación de la educación superior ecuatoriana debería concretarse en los tres puntos que he mencionado, y en muchos otros que no he podido desarrollar: a) la consideración del desarrollo integral y sostenible como horizonte de sentido para la educación superior y sus tres funciones sustantivas; b) la comprensión del conocimiento como un bien público y de la educación superior como un derecho y deber de la sociedad civil, un servicio tanto estatal como civil, al tiempo que se defiende la diversidad de ofertas de sentido en educación, porque diversa es la sociedad ecuatoriana; c) la inclusión educativa como dinámica que reúne calidad y acceso democrático a la educación superior.

Ojalá que las universidades católicas, y también todas las universidades ecuatorianas fueran reconocidas no por el número de estudiantes que excluyen sino por la excelencia en conocimientos y contribución a la vida del planeta que son capaces de suscitar en quienes asisten a sus aulas. 


\section{BIBLIOGRAFÍA}

Benedicto XVI. (2009). Carta Encíclica Caritas in veritate. Sobre el desarrollo humano integral en la caridad y la verdad.

Caamaño López, J. (septiembre de 2016). La "ecología integral" de la Encíclica Laudato Si. Sal Terrae, 104/8(1214), 679-692.

Concilio Vaticano II. (1965). Gaudium et Spes. Constitución pastoral sobre la Iglesia en el mundo actual.

Concilio Vaticano II. (1965b). Declaración Gravissimum Educationis sobre la educación cristiana.

De La Fuente, J. (2008). Sociedad del conocimiento y la universidad. Educación Superior y Sociedad, 13(1. Transformaciones sociales y desafíos universitarios en América Latina), 21-31.

Dias Sobrinho, J. (2008). Calidad, pertinencia y responsabilidad social de la universidad latinoamericana y caribeña. En A. Gazzola, \& A. Didriksson, Tendencias de la Educación Superior en América Latina y el Caribe (págs. 87-112). Caracas: IESALC-UNESCO.

Francisco. (2015). Carta Encíclica Laudato Si. Sobre el cuidado de la casa común. Buenos Aires: Conferencia Episcopal Argentina.

Francisco. (7 de julio de 2015b). Encuentro con el mundo de la enseñanza. Discurso del Santo Padre. Recuperado el 2 de octubre de 2015, de http://w2.vatican.va/content/francesco/es/speeches/2015/july/documents/papa-francesco_20150707_ecuador-scuola-universita.html

Juan Pablo II. (1991). Carta Encíclica Centesimus Annus. En el centenario de la "Rerum Novarum". Juan Pablo II. (1991). Constitución Apostólica Ex Corde Ecclesiae sobre las universidades católicas. Laville, J.-L., \& Cattani, A. D. (Edits.). (2006). Dictionnaire de l'autre économie. Paris: Desclée de Brouwer.

Ricossa, S. (2000). Diccionario de economía. México, D.F.: Siglo XXI Editores.

Sen, A. (1999). Development as Freedom. Oxford: Oxford University Press.

Stiglitz, J. E. (1999). Knowledge as a Global Public Good. En I. Kaul, I. Grunberg, \& M. A. Stern, Global Public Goods. International Cooperation in the 21st Century (págs. 308-327). New York: UNDP / Oxford University Press. 
UNESCO / Conferencia Mundial sobre Educación Superior 2009. (2010). La nueva dinámica de la educación superior y la investigación para el cambio social y el desarrollo. París: UNESCO.

V Conferencia General del Episcopado Latinoamericano y del Caribe. (2007). Documento conclusivo. Aparecida.

Zamagni, S. (diciembre de 2007). El bien común en la sociedad postmoderna: propuestas para la acción político-económica. Revista Cultura Económica, XXV(70), 23-43. 